\title{
Aphid Protein Effectors Promote Aphid Colonization in a Plant Species-Specific Manner
}

\author{
Marco Pitino and Saskia A. Hogenhout \\ Cell and Developmental Biology, The John Innes Centre, Norwich Research Park, Norwich, NR4 7UH, U.K.
}

Submitted 10 July 2012. Accepted 16 September 2012.

\begin{abstract}
Microbial pathogens and pests produce effectors to modulate host processes. Aphids are phloem-feeding insects, which introduce effectors via saliva into plant cells. However, it is not known if aphid effectors have adapted to modulate processes in specific plant species. Myzus persicae is a polyphagous insect that colonizes Arabidopsis thaliana and Nicotiana benthamiana, while the pea aphid Acyrthosiphon pisum specializes on colonizing plant species of the family Fabaceae. We found that $M$. persicae reproduction increased on transgenic Arabidopsis, producing the $M$. persicae effectors C002, PIntO1 (Mp1), and PIntO2 (Mp2), whereas reproduction of $M$. persicae did not increase on Arabidopsis producing the $A$. pisum orthologs of these three proteins. Plant-mediated RNA interference experiments showed that $\mathrm{c002}$ - and $\mathrm{PIntO2}$-silenced $\mathrm{M}$. persicae produce less progeny on Arabidopsis and $N$. benthamiana than nonsilenced aphids. Orthologs of c002, PIntO1, and PIntO2 were identified in multiple aphid species with dissimilar plant host ranges. We revealed high nonsynonymous versus synonymous nucleotide substitution rates within the effector orthologs, indicating that the effectors are fast evolving. Application of maximum likelihood methods identified specific sites with high probabilities of being under positive selection in PIntO1, whereas those of $\mathrm{CO02}$ and PIntO2 may be located in alignment gaps. In support of the latter, a $M$. persicae c002 mutant without the NDNQGEE repeat region, which overlaps with an alignment gap in $\mathbf{C 0 0 2}$, does not promote $M$. persicae colonization on Arabidopsis. Taken together, these results provide evidence that aphid effectors are under positive selection to promote aphid colonization on specific plant species.
\end{abstract}

Insect pests are often highly selective in their host choices for long-term feeding or reproduction. The majority of chewing herbivores such as lepidopterans and sap-feeding insects of the order Hemiptera are able to colonize single or a few related plant species and, as such, are described as oligophagous (Schoonhoven et al. 2005). Relatively few insect species are polyphagous, with wide host ranges consisting of plant species

Current address for M. Pitino: United States Horticulture Research Laboratory, United States Department of Agriculture-Agricultural Research Service, Fort Pierce, FL 34945, U.S.A.

Corresponding author: S. A. Hogenhout; Telephone: +44 1603 450393; Fax: +44 1603 450045; E-mail: saskia.hogenhout@jic.ac.uk

* The $\boldsymbol{e}$-Xtra logo stands for "electronic extra" and indicates that seven supplementary tables and two supplementary figures are published online.

(C) 2013 The American Phytopathological Society of multiple distantly related plant families. There is a growing body of evidence that insects produce virulence factors (effectors) that modulate host processes to facilitate feeding and colonization (Hogenhout and Bos 2011). Given that the majority of insects are oligophagous (Schoonhoven et al. 2005), pest effectors are likely to act in a host-specific manner (Hogenhout and Bos 2011). However, few investigations have focused on the host-specific nature of insect effector action. This research is important, because targeting of pest effectors by vaccination of humans and animals and for selection for resistance in plants could limit insect colonization and insect-transmitted diseases.

The sap-feeding aphids (family Aphididae, order Hemiptera) are particularly amenable for studying the role of effectors in insect-host interactions (Dogimont et al. 2010; Hogenhout and Bos 2011). The genome of the legume-specialist pea aphid Acyrthosiphon pisum has been sequenced (IAGC 2010) and transcriptome data of a number of aphid species, including the polyphagous green peach aphid Myzus persicae, are available (Ollivier et al. 2012; Ramsey et al. 2007). Moreover, aphid effectors have been identified (Bos et al. 2010; Mutti et al. 2006, 2008; Pitino et al. 2011; Will et al. 2007). Because $M$. persicae utilizes the model plants Nicotiana benthamiana and Arabidopsis thaliana as feeding and reproductive hosts, the role of effectors in aphid-plant interactions can be studied directly on the plant host by expressing $M$. persicae effector genes transiently in $N$. benthamiana leaves and stably in Arabidopsis and by silencing effector-encoding genes in $M$. persicae by plant-mediated RNA interference (RNAi) (Bos et al. 2010; Pitino et al. 2011). In addition, aphids have a short generation time and can build up to large populations consisting of genetically identical asexually producing females, enabling the consistent and accurate monitoring of effector influence on aphid colonization ability in relatively short time frames.

Aphids most likely introduce effectors via their saliva during feeding (Cherqui and Tjallingii 2000; Miles 1999; Tjallingii 2006). Aphids have piercing-sucking mouthparts that consist of stylets which can navigate between plant cells to the vascular tissue, where they feed mainly from the phloem. Aphids produce several types of saliva (Tjallingii 2006). Gelling sheath saliva surrounds the aphid stylets, protects these aphid mouthparts from the apoplastic defenses, and reduces the influx of extracellular compounds into plant cells. Watery saliva is released into plant cells during probing and penetrations while navigating to the vascular system and during long-term feeding from the plant phloem. Mass spectrometry of proteins in aphid saliva and salivary glands revealed a diversity of proteins, and some of these proteins appear to have effector functions (Carolan et al. 2009, Harmel et al. 2008; Nicholson et al. 2012). Conserved proteins among insects, such as glucose oxidase (GOX), elicit plant defense responses (Tian et al. 2012). 
Furthermore, calcium-binding proteins prevent phloem sieve cell plugging upon mechanical damage by the aphid stylets (Will et al. 2007, 2009). In addition, the aphid-specific protein C002 aids aphid colonization. Silencing of $\mathrm{c002}$ by injection of double-stranded (ds)RNA into the Acyrthosiphon pisum hemolymph or by feeding $M$. persicae on transgenic plants expressing dsRNA corresponding to $\mathrm{C} 002$ by plant-mediated RNAi reduces survival or reproduction rates of these aphids (Mutti et al. 2006; Pitino et al. 2011). C002 was detected in A. pisum-exposed plants (Mutti et al. 2008) and expression of $M$. persicae $\mathrm{C} 002$ in plants promotes $M$. persicae reproduction rates (Bos et al. 2010). Finally, the $M$. persicae effector Mp10 suppresses basal immunity pathways but also triggers an SGT1dependent chlorosis response in $N$. benthamiana (Bos et al. 2010). Together, these data provided evidence that aphid effectors are likely to suppress plant defense to aphids, modulate plant processes to aid aphid colonization, or may be recognized by specific plant resistance proteins, resulting in effectortriggered immunity (Hogenhout and Bos 2011).

In this study, we wished to elucidate whether effectors act in a plant-species-specific manner, thereby providing evidence that aphid effectors may play a role in determining aphid host range. We report here that $M$. persicae has more progeny on transgenic Arabidopsis that produce M. persicae $\mathrm{C} 002$ but not on those that produce the A. pisum $\mathrm{C} 002$ ortholog or an $M$. persicae $\mathrm{C} 002$ mutant in which the sequence corresponding to a polymorphic repeat was deleted. Similar results were obtained for two other $M$. persicae and A. pisum effector orthologs. These results indicate that $M$. persicae effectors modulate Arabidopsis responses to the benefit of $M$. persicae, whereas the $A$. pisum effectors do not. These data are consistent with Arabidopsis being a host for $M$. persicae but not for A. pisum. We determined that aphid effectors are fast evolving and identified sites under positive selection.

\section{RESULTS}

\section{M. persicae $\mathrm{C002}$ promotes M. persicae colonization on Arabidopsis.}

Peptides corresponding to $\mathrm{C} 002$ were detected in the saliva and salivary glands of $M$. persicae (EC389283; Harmel et al. 2008) and A. pisum (Mutti et al. 2008) (ACYPI008617; Carolan et al. 2011). These proteins possess signal peptides with $\geq 0.925$ probability values and predicted cleavage sites between amino acids 24 and 25 (Fig. 1A). We previously noted that $M$. persicae $\mathrm{CO02}$ (MpC002) contains five repeats with the sequence NDN QGEE in the N-terminal part of the protein that are lacking in $A$. pisum C002 (ApC002) (Bos et al. 2010) (Fig. 1A). To assess whether the sequence divergence between the two proteins determines effector specificity, we generated transgenic Arabidopsis Col-0 lines stably expressing transcripts corresponding to MpC002 and ApC002 under control of the Arabidopsis sucrose transporter promoter AtSuc2 for phloem-specific expression. We generated three independent transgenic lines foreach protein, named AtSuc2:MpC002 lines 4, 6, and 7 and AtSuc2:ApC002 lines 3, 4, and 8. A transgenic line (AtSuc2:GFP) that stably expresses the green fluorescent protein (GFP) coding sequence and Col- 0 were included as controls. Transgene transcripts were detected in all Arabidopsis lines (Supplementary Fig. S1A).

$M$. persicae produced approximately $20 \%$ more progeny on AtSuc2:MpC002 lines than on Col-0 and AtSuc2:GFP plants (generalized linear model [GLM], three biological replicates with $n=4$ plants per replicate, $P<0.05$ ) (Fig. 1B). In contrast, $M$. persicae reproduction did not increase on the AtSuc2: ApC002 lines (GLM, $P=$ not significant [ns]) (Fig. 1B) even though ApC002 transcript levels were similar to the $\mathrm{MpC002}$ transcript levels of AtSuc2:MpC002 lines 4 and 6.
To assess whether the MpC002 NDNQGEE repeat (Fig. 1A, motifs I to V), which is lacking from ApC002 (Fig. 1A), is responsible for the $M$. persicae progeny increase, we also generated three transgenic Col-0 lines, which produce a $\mathrm{MpC002}$ mutant without the five NDNQGEE repeats (MpC002 $\Delta$ ) (Fig. 1A) under control of the AtSuc2 promoter. The $M$. persicae reproduction rates on the AtSuc2: MpC002 $\Delta$ lines did not increase and were similar to those of the aphids on AtSuc2: ApC002 plants (GLM, $P=$ ns) (Fig. 1B), even though the $\mathrm{MpC} 002$ and $\mathrm{MpC} 002 \Delta$ transcript levels were similar. Together, these data indicate that the five NDNQGEE present in MpC002 are involved in promoting $M$. persicae reproduction on Arabidopsis.

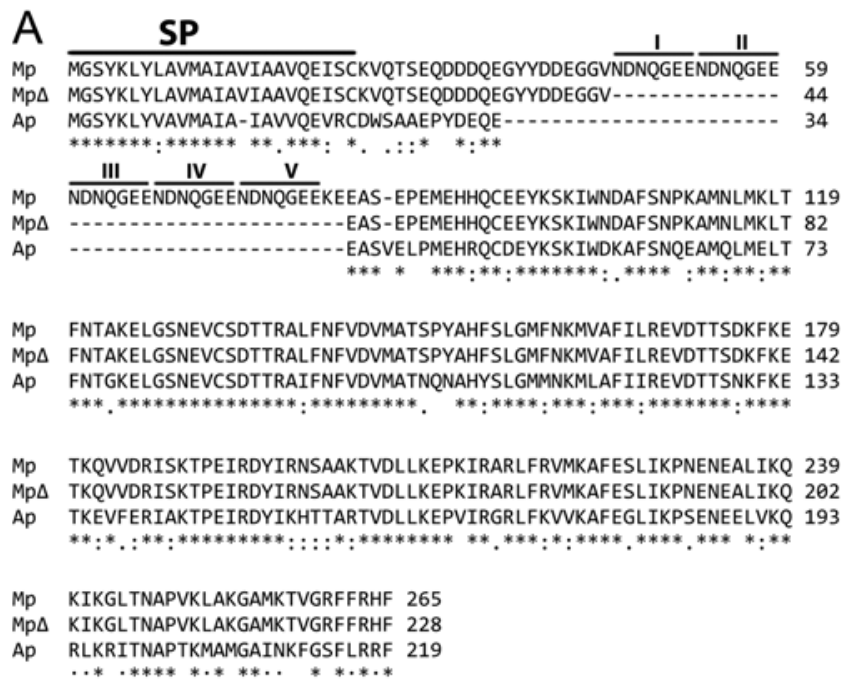

B

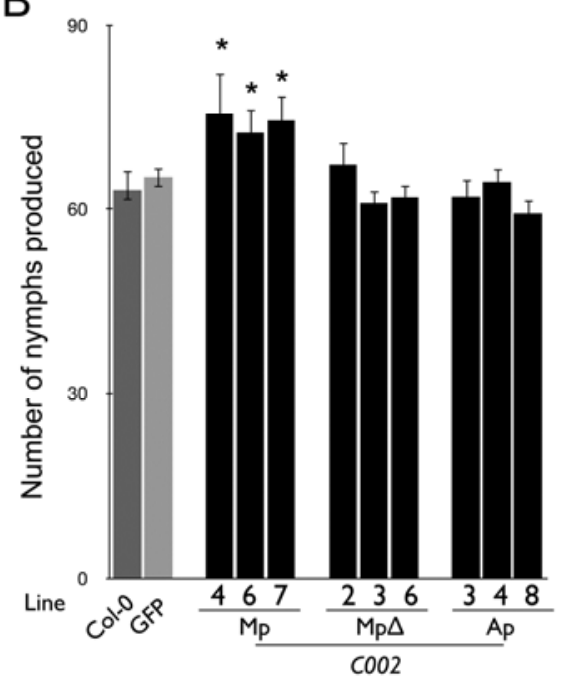

Fig. 1. Sequence polymorphisms of C002 confer effector specificity. A, Myzus persicae (Mp) C002 contains a region with five times the NDNQGEE sequence lacking in Acyrthosiphon pisum (Ap) C002 and is deleted from the $\mathrm{MpC} 002 \Delta$ mutant $(\mathrm{Mp} \Delta)$ of $M$. persicae $\mathrm{C} 002$. NDNQGEE repeats are numbered $\mathrm{I}$ to $\mathrm{V}$ and $\mathrm{SP}=$ signal peptide. $\mathbf{B}, M$. persicae produces more progeny on Arabidopsis MpC002 transgenic lines but not on the ApC002 and MpC002 $\Delta$ lines. Single plants were seeded with five nymphs, which started to produce offspring approximately on day 10. Progeny were counted on days 10,13 , and 16 and removed. Columns show the average nymph production plus standard deviations of three biological replicates, with each replicate consisting of the progeny produced by 20 aphids at 5 aphids per plant ( $n=4$ plants). Numbers under columns on $x$ axis indicate independent transgenic lines. Asterisk (*) indicates generalized linear model, $P<0.05$ compared with green fluorescent protein plants. 


\section{M. persicae PIntO1 and PIntO2 promote \\ M. persicae colonization on Arabidopsis.}

We previously detected 46 candidate effectors in the $M$. persicae salivary gland transcriptome (Bos et al. 2010). Of these candidate effectors, Mp1 has a signal peptide with probability score of 0.964 and a predicted cleavage site between amino acids 19 and 20 (Fig. 2A), and peptides corresponding to Mp1 were found in mass spectrometry analysis of $M$. persicae saliva (DW011417, EE264598; Harmel et al. 2008). MpC002 and Mp1 have similar expression levels in M. persicae (Table 1). To assess whether Mp1 is an effector, we generated transgenic Arabidopsis Col-0 lines producing Mp1 under control of the AtSuc2 promoter generating transgenic lines 2, 6, and 7 . $M$. persicae reproduction rates on these lines were overall higher (GLM, three biological replicates with $n=4$ plants per replicate, $P<0.05)$ than on AtSuc2:GFP plants, with those on line 2 being more variable (Fig. 2B). This is consistent with lines 6 and 7 having higher Mp1 transcript levels than line 2. Because of the positive effect of $\mathrm{Mp} 1$ on $M$. persicae progeny production, we renamed this protein "Progeny Increase to Overexpression 1" (PIntO1).

We identified homologs of $M$. persicae PIntO1 (MpPIntO1) in A. pisum (ApPIntO1). These proteins have predicted signal peptides with probability score of 0.999 and cleavage sites between amino acids 19 and 20 (Fig. 2A). ApPIntO1 peptides were identified in A. pisum saliva (APG01142; Carolan et al. 2009) and salivary glands by mass spectrometry analysis

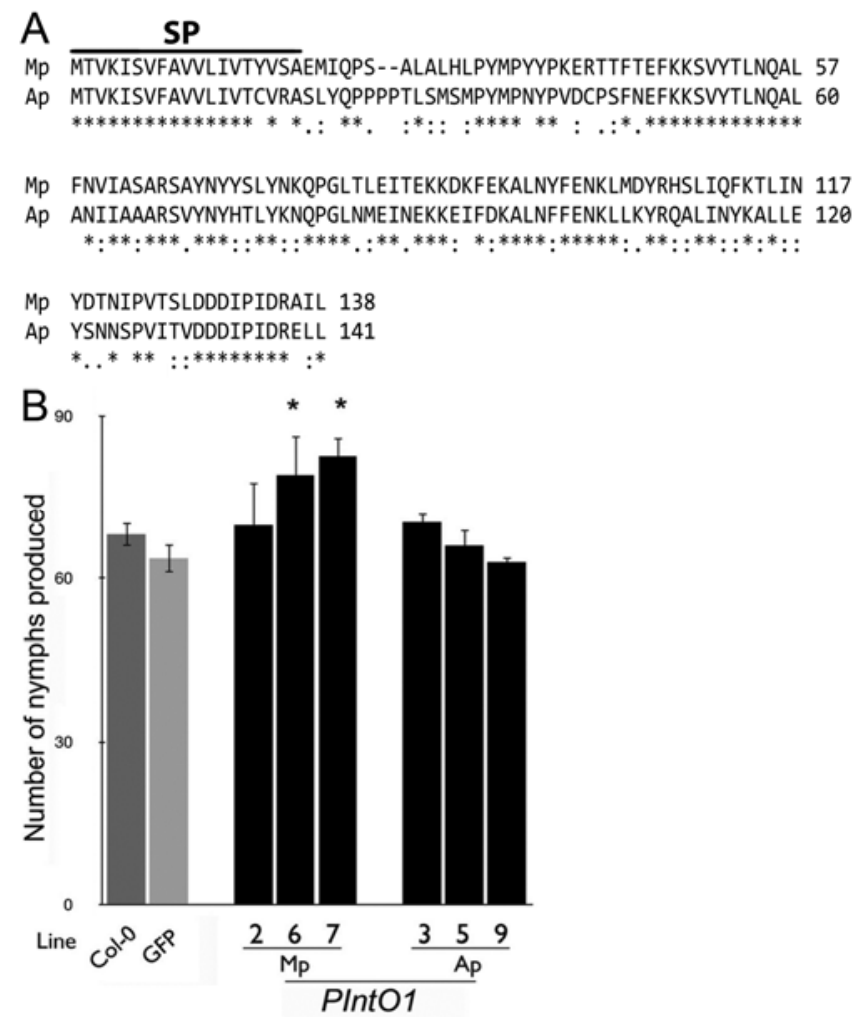

Fig. 2. MpPIntO1 (Mp1) but not ApPIntO1 promotes aphid colonization on Arabidopsis. A, Protein alignment of Myzus persicae and Acyrthosiphon pisum PIntO1. B, M. persicae produces more progeny on Arabidopsis MpPIntO1 (Mp1) transgenic lines but not on the ApPIntO1 lines. Single plants were seeded with five nymphs, which started to produce offspring approximately on day 10 . Progeny were counted on days 10,13 , and 16 and removed. Columns show the average nymph production plus standard deviations of three biological replicates, with each replicate consisting of the progeny produced by 20 aphids at 5 aphids per plant $(n=4$ plants). Numbers under columns on $x$ axis indicate independent transgenic lines. Asterisk $(*)$ indicates generalized linear model, $P<0.05$ compared with green fluorescent protein plants.
(APG01142; Carolan et al. 2011). M. persicae reproduction assays on three independent ApPIntO1 transgenic lines revealed that the $A$. pisum effector does not promote $M$. persicae reproduction (Fig. 2B) even though the MpPIntO1 and ApPIntO1 transcript levels were similar.

We wished to investigate whether less abundant effector proteins in aphid salivary glands promote aphid colonization on plants. Mp2 was previously identified as a candidate effector based on the presence of a signal peptide sequence and expression in M. persicae salivary glands (Bos et al. 2010). Mp2 has a signal peptide with probability score of 0.990 and a predicted cleavage site between amino acids 20 and 21 (Fig. 3A). Mp2 transcript levels are threefold lower than those corresponding to $\mathrm{MpC002}$ and $\mathrm{PIntO} 1$ in $M$. persicae (Table 1). M. persicae produced more progeny on three independent lines producing Mp2 under control of the AtSuc2 promoter compared with AtSuc2:GFP plants (Fig. 3B) in which those on lines 1 and 2

Table 1. Expression levels of three Myzus persicae candidate effector genes

\begin{tabular}{lc}
\hline Gene & Fold expression compared with $\boldsymbol{\beta}$-tubulin \\
\hline CO02 & $3.02(0.47)$ \\
Mp1 (PIntO1) & $2.96(0.42)$ \\
Mp2 (PIntO2) & $0.01(0.02)$ \\
\hline
\end{tabular}

${ }^{a}$ Mean expression levels of three biological replicates. Numbers in parentheses represent standard deviation.

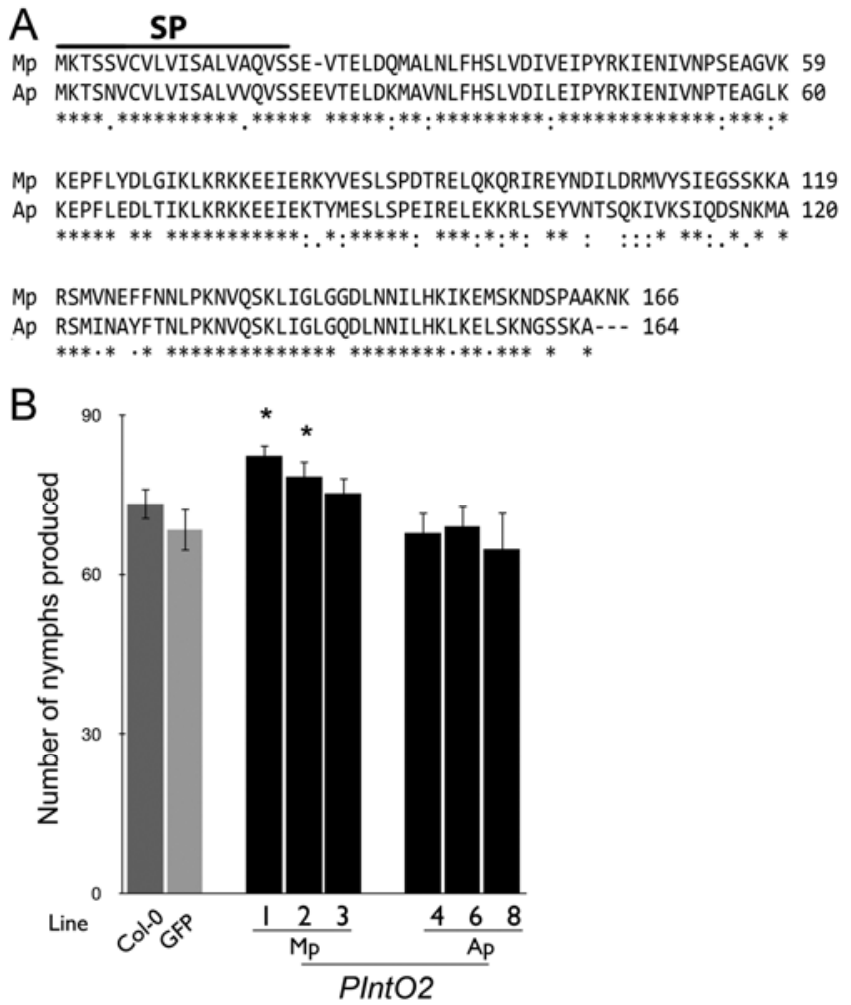

Fig. 3. $\mathrm{MpPIntO} 2(\mathrm{Mp} 2)$ but not $\mathrm{ApPIntO} 2$ promotes aphid colonization on Arabidopsis. A, Protein alignment of Myzus persicae and Acyrthosiphon pisum $\mathrm{PIntO2}$. B, M. persicae produces more progeny on Arabidopsis $\mathrm{MpPIntO} 2(\mathrm{Mp} 2)$ transgenic lines but not on the ApPIntO2 lines. Single plants were seeded with five nymphs, which started to produce offspring approximately on day 10 . Progeny were counted on days 10,13 , and 16 and removed. Columns show the average nymph production plus standard deviations of three biological replicates, with each replicate consisting of the progeny produced by 20 aphids at 5 aphids per plant $(n=4$ plants). Numbers under columns on $x$ axis indicate independent transgenic lines. Asterisk $(*)$ indicates generalized linear model, $P<0.05$ compared with green fluorescent protein plants. 
were higher (GLM, three biological replicates with $n=4$ plants per replicate, $P<0.05$ ) than on line 3 , consistent with higher expression levels of the Mp2 transcripts in lines 1 and 2 compared with line 3 . Hence, we named Mp2 "Progeny Increase to Overexpression 2" (PIntO2). We found a homolog of $\mathrm{PIntO} 2$ in $A$. pisum that has a signal peptide with probability score of 0.997 and a predicted cleavage site between amino acids 20 and 21 (Fig. 3A). However, M. persicae did not produce more progeny on three independent AtSuc2:ApPIntO2 transgenic lines (Fig. 3B), which all have transgene expression levels similar to the AtSuc2:ApPIntO2 lines. Thus, M. persicae PIntO1 and $\mathrm{PIntO} 2$ promote $M$. persicae reproduction on Arabidopsis, whereas A. pisum PIntO1 and PIntO2 do not.

\section{Silencing of PIntO1 and PIntO2 reduces aphid reproduction on plants.}

To obtain additional evidence that PIntO1 and $\mathrm{PIntO} 2$ have effector functions in $M$. persicae, we wished to silence the expression of corresponding genes in $M$. persicae using plantmediated RNAi and assess whether this reduced $M$. persicae reproduction, as has been shown for MpC002-silenced aphids (Pitino et al. 2011). Nucleotide sequences corresponding to amino acids MEMIQP to IDRAIL of PIntO1 and amino acids EVTEL to SKNDSP of PIntO2 were cloned as inverted repeats into the Agrobacterium tumefaciens pJawohl8-RNAi binary silencing vector (I. E. Somssich) and transiently expressed in $N$. benthamiana leaves by Agrobacterium-mediated infiltration (agro-infiltration) (Pitino et al. 2011). Aphid reproduction rates were analyzed and silencing of aphid genes were assessed as previously described (Pitino et al. 2011). C002-silenced aphids produced fewer nymphs compared with control aphids fed on $N$. benthamiana producing double-stranded GFP (analysis of
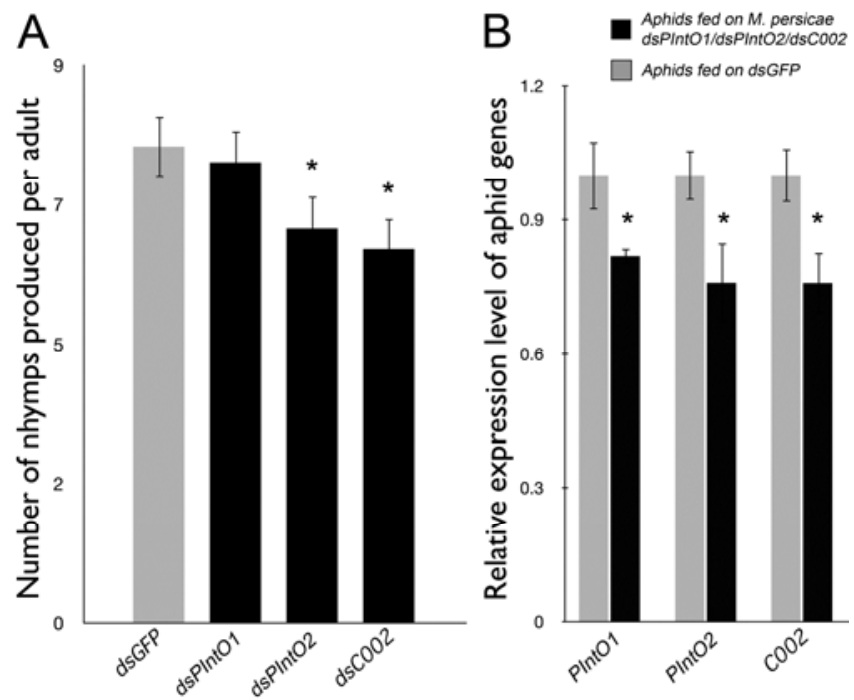

Fig. 4. Silencing of Myzus persicae $\mathrm{C} 002$ and $\mathrm{PIntO} 2$ on Nicotiana benthamiana reduces aphid fecundity. A, $\mathrm{C} 002$ and $\mathrm{PIntO} 2$-silenced $M$. persicae produce fewer progeny on $N$. benthamiana. Individual leaf disks were seeded with four nymphs, which started to produce offspring approximately on day 12. Progeny was counted on days 12, 14 and 17 and removed. Columns show the average nymph production plus standard deviation per aphid of 6 biological replicates with each replicate consisting of $n=4-6$ leaf disks. Asterisk $(*)$ indicates analysis of variance, $P<0.05$. B, $M$. persicae exposed to leaf disks in A have reduced C002, PIntO1, and PIntO2 expression levels. Columns indicate the average target gene $(x$ axis) expression levels and standard deviations relative to tubulin and L27 reference genes (Pitino et al. 2011) of $n=3$ biological replicates. In each biological replicate, the four nymph-producing adults on leaf disks of three of six experiments shown in A were collected at day 17 and pooled for RNA extraction and quantitative reverse-transcriptase polymerase chain reaction. Asterisk (*) indicates Student's $t$ test, $P<0.05$. variance [ANOVA], six biological replicates with $n=4$ to 6 leaf disks per replicate, $P=0.009$ ) (Fig. 4A), confirming previous results (Pitino et al. 2011). Silencing of PIntO2 in $M$. persicae also reduced nymph production (ANOVA, $P=0.041$ ), while silencing of $M$. persicae PIntO1 did not (ANOVA, $P=$ 0.732) (Fig. 4A). The expression levels of the effector genes were similarly reduced in the silenced aphids (Student's $t$ test, three biological replicates, $P<0.05$ ) (Fig. 4B).

We also assessed the effect of silencing of three effector genes on Arabidopsis. We used the published silencing lines
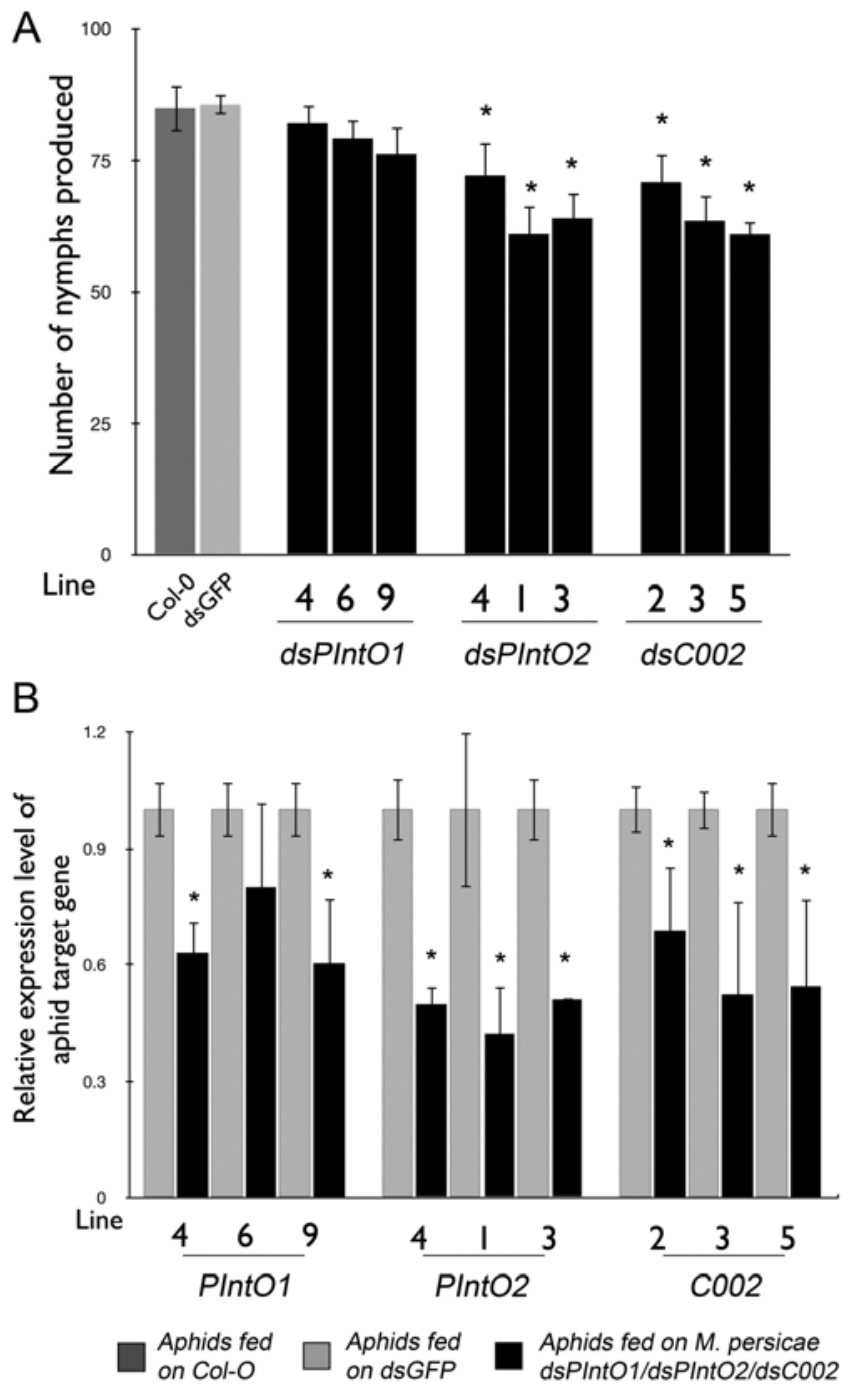

Fig. 5. Silencing of Myzus persicae $\mathrm{C} 002$ and $\mathrm{PIntO} 2$ on Arabidopsis reduces aphid fecundity. A, C002- and PIntO2-silenced $M$. persicae produce fewer progeny on Arabidopsis. Single plants were seeded with five nymphs, which started to produce offspring approximately on day 10 . Progeny were counted on days 10, 13, and 16 and removed. Columns show the average nymph production plus standard deviations of three biological replicates, with each replicate consisting of the progeny produced by 20 aphids at 5 aphids per plant ( $n=4$ plants). Asterisk (*) indicates generalized linear model, $P<0.05$ compared with aphids fed on doublesranded green fluorescent protein (dsGFP) plants. B, M. persicae exposed to transgenic plants in A have reduced $\mathrm{C} 002$, PIntO1, and PIntO2 expression levels. Target gene expression levels of experimental plants and dsGFP control plants were assayed independently for each line as shown. Columns indicate the average target gene ( $x$ axis) expression levels and standard deviations relative to tubulin and L27 reference genes (Pitino et al. 2011) of $n=3$ biological replicates. In each biological replicate, the five nymph-producing adults kept on four plants for 16 days of the experiment shown in A were pooled for RNA extraction and quantitative reversetranscriptase polymerase chain reaction. Asterisk (*) indicates Student's $t$ test, $P<0.05$ compared with aphids fed on dsGFP plants. 
for MpC002 (Pitino et al. 2011) and generated three independent silencing lines for PIntO1 and PIntO2. Plant-mediated RNAi of both $\mathrm{MpC} 002$ and $\mathrm{PIntO} 2$ genes reduced $M$. persicae nymph production (GLM, three biological replicates with $n=$ 4 plants per replicate, $P<0.05$ ), whereas silencing of PIntO1 did not (GLM, $P>0.05$ ) (Fig. 5A). With the exception of aphids fed on dsPIntO1 line 6, the expression levels of target genes in $M$. persicae were significantly reduced on all lines (Student's $t$ test, three biological replicates, $P<0.05$ ) (Fig. 5B). Together, these results show that plant-mediated silencing of the M. persicae $\mathrm{C} 002$ and $\mathrm{PIntO} 2$ genes on $N$. benthamiana and Arabidopsis reduced nymph production, whereas silencing of the PIntO1 gene did not.

\section{Assessment of positively selected sites in C002, PIntO1, and PIntO2.}

The differential effects of the M. persicae and A. pisum effectors may reflect the host ranges of these aphid species, because M. persicae survives and reproduces readily on Arabidopsis and other members of the family Brassicaceae, whereas A. pisum does not survive on these plant species. Genes functionally important in environmental adaptation are often subject to positive selection. An indicator of protein-coding DNA sequences under positive selection pressure is a higher nonsynonymous $\left(d_{N}\right)$ versus a synonymous $\left(d_{S}\right)$ nucleotide substitution rate, resulting in ratio $\omega=d_{N} / d_{S}>1$, indicating that sequences are fast evolving and identification of sites that are under high probability of positive selection (Ina 1995; Li et al. 1985; Nei and Gojobori 1986; Yang and Bielawski 2000). We assessed if the C002, PIntO1 and PIntO2 effectors are subject to positive selection pressures.

First, we searched the GenBank expressed sequence tags (EST) database for aphid EST corresponding to C002, PIntO1, and PIntO2 and selected full-length transcripts. C002 EST in- cluded 29 from $M$. persicae, 100 from $A$. pisum, and 15 from Aphis gossypii (Supplementary Table S2) and deduced protein sequences harbored predicted $\mathrm{N}$-terminal signal peptides with high probability scores (Supplementary Table S1). The $29 \mathrm{M}$. persicae EST consist of two copies of MpC002_a (20 EST) and MpC002_b (9 EST). MpC002_a has the same sequence as the $\mathrm{MpC002}$ sequence used in the aphid reproduction assays (Fig. 1A). MpC002_b is almost identical in sequence to MpC002_a, except for the absence of one NDNQGEE repeat corresponding to amino acids 46 to 51 of MpC002_a (Fig. 6A). Acyrthosiphon pisum and Aphis gossypii both lack the five NDNQGEE repeats and have other sequence differences compared with MpC002 as well (Fig. 6A). The phylogeny of C002 sequences is consistent with the aphid phylogeny based on 203 concatenated nuclear genes (Ollivier et al. 2012). indicating that the genes are orthologs (Supplementary Fig. S2). The pairwise $d_{N} / d_{S}$ ratios of the $\mathrm{C} 002$ sequences between aphid species are high, ranging from 0.536 to 0.617 (Supplementary Table S3), which is well above the mean $d_{N} / d_{S}$ values of 0.064 to 0.094 based on a core set of 253 reciprocal best host genes shared by aphid species (Ollivier et al. 2010), indicating that C002 is fast evolving. To identify specific sites under positive selection, we examined null and alternative maximum likelihood (ML) models of codon substitutions of M1 versus M2, M0 versus M3, and M7 versus M8 and examined their $\chi^{2}$ distributions (Nielsen and Yang 1998; Yang and Bielawski 2000). None of these model comparisons revealed significant evidence that specific $\mathrm{C} 002$ amino acids residues are under positive selection $(P$ value $\geq 0.14$ ) (Supplementary Table $S 4)$. These results confirm previous findings (Ollivier et al. 2010). However, the ML models exclude gaps in alignments that overlie the NDN QGEE repeats (Fig. 6A). Therefore, diversifying selection may act on sites located in the alignment gaps.

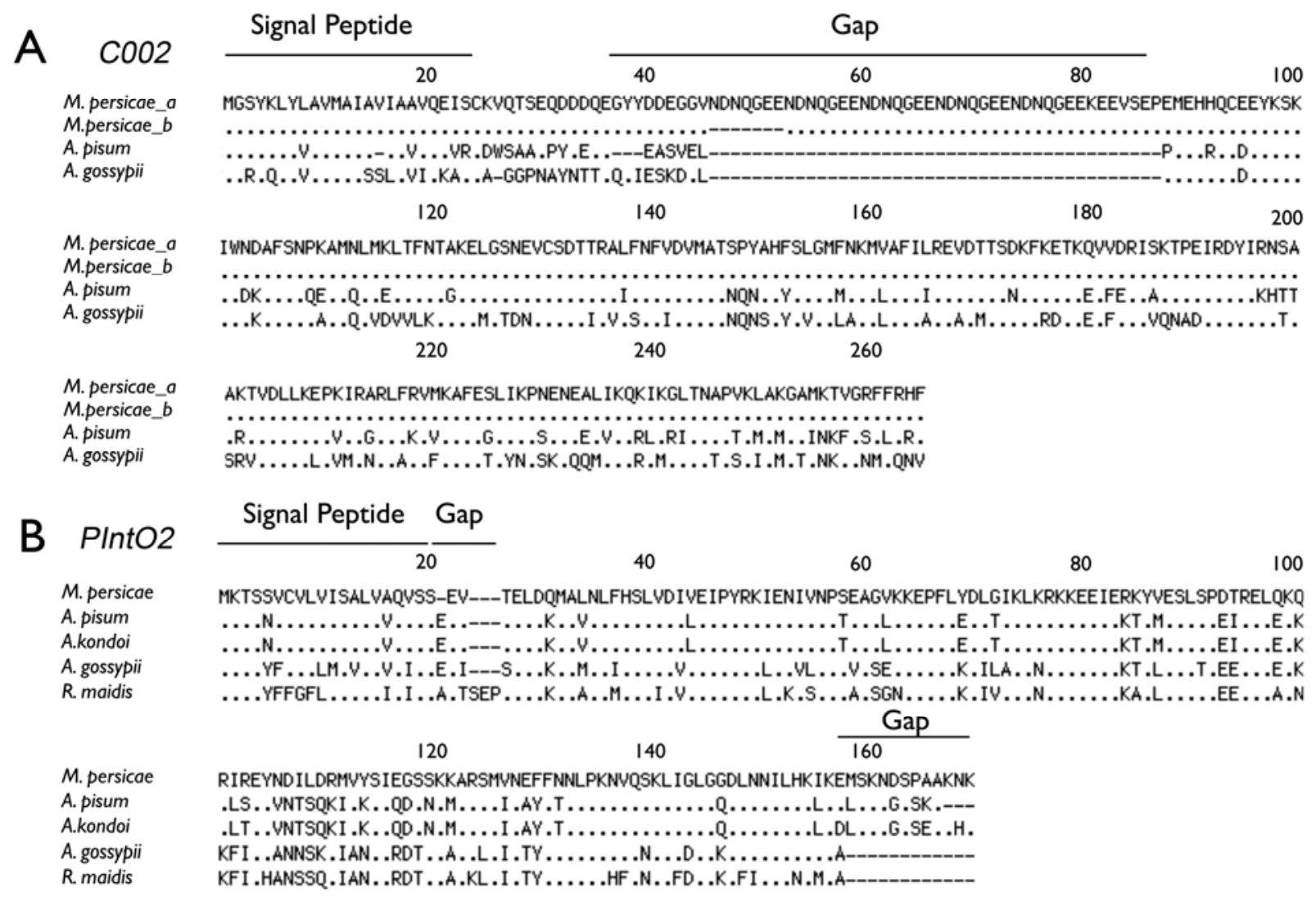

Fig. 6. Alignments of $\mathbf{A}, \mathrm{C} 002$ and $\mathbf{B}, \mathrm{PIntO} 2$ orthologs of aphid species reveal single-amino-acid and gap polymorphisms. Alignments were generated in Muscle (v3.7). Regions corresponding to the signal peptides and gaps are indicated above the alignments. 
We also examined the molecular evolution of PIntO1 and PIntO2 orthologs. Full-length PIntO1 were identified in several aphid species, including 44 in $M$. persicae and 80 in Acyrthosiphon pisum. The A. pisum EST group into PIntO1_a (36 EST) and PIntO1_b (44 EST) sequences. Of these, PIntO1_a was used for generating the transgenic plants and conducting $M$. persicae fecundity assays (Fig. 2). PIntO2 EST were identified in five aphid species, with 16 and 36 EST in M. persicae and A. pisum, respectively. Deduced protein sequences of PIntO1 and PIntO2 EST all harbor predicted Nterminal signal peptides with high probability scores.

The PIntO1 alignment showed two areas with gaps of 2 to 3 amino acids immediately after the signal peptide sequences (Fig. 7), whereas the $\mathrm{PIntO} 2$ alignment revealed several gaps in some sequences, including immediately after the signal peptide sequence and at the end of the proteins (Fig. 6B). The phylogenetic trees of these effectors were consistent with that of the aphid phylogeny based on 203 nuclear genes, indicating that they are orthologs (Ollivier et al. 2012). The pairwise $d_{N} / d_{S}$ ratios of the PIntO1 and $\mathrm{PIntO} 2$ were higher overall than the 0.064 to 0.094 average (Ollivier et al. 2010), ranging from 0.375 to 1.038 for PIntO1 and 0.413 to 0.896 for PIntO2, though the A. pisum and A. kondoi pairwise comparison of PIntO2 was lower at 0.067 . This indicates that both PIntO1 and $\mathrm{PIntO} 2$ are fast evolving among aphid species. The ML models of codon substitutions identified significant $\chi^{2}$ distributions for PIntO1 in all models $(P$ value $\leq 0.0069)$ (Supplementary Table S5). Positively selected sites were identified from $70 \%$ to greater than $99 \%$ (Fig. 7). On the other hand, no consistently significant $\chi^{2}$ distributions among the models were identified for PIntO2 (Supplementary Table S6). However, positive selection may act on sites in alignment gaps that are excluded from the ML model analyses. Altogether, these data suggest that specific PIntO1 sites are subject to positive selection, whereas such sites were not identified in C002 and PIntO2, though positive selection may act on sites located in alignment gaps of the latter two effectors.

\section{DISCUSSION}

Aphids are phloem-feeding insects which release saliva that contains effectors into plant cells. Evidence that $\mathrm{C} 002$ is an effector in M. persicae and A. pisum has accumulated (Bos et al.
2010; Mutti et al. 2006, 2008; Pitino et al. 2011). This study is in agreement with these published findings, because we found that $M$. persicae produces more progeny on transgenic plants that produce $\mathrm{MpC002}$ and less progeny on plants that induce silencing of $\mathrm{c002}$ in $M$. persicae. This indicates that $\mathrm{C} 002$ functions in the plant host upon release by the aphid consistent with effectors functioning in hosts rather than in the parasites in which they are synthesized (Hogenhout et al. 2009).

Although there is evidence that aphids produce effectors that modulate plant host processes to the benefit of aphids, it is not yet clear whether these effectors are involved in determining aphid compatibility with the plant host. M. persicae has a broad host range consisting of plant species in multiple plant families, and this includes Arabidopsis and $N$. benthamiana. However, the host range of $A$. pisum is restricted to plant species in the Fabaceae (Leguminosae) family. We observed that $M$. persicae and A. pisum effector orthologs harbor gap and single amino acid polymorphisms, which we hypothesized may reflect their function in different plant species. In this study, we have provided evidence for this hypothesis, because $M$. persicae generates more progeny on transgenic Arabidopsis plants that produce the $M$. persicae effectors C002, PIntO1, and $\mathrm{PIntO} 2$ but not on plants that produce the A. pisum orthologs of these proteins. Thus, the $M$. persicae protein effectors apparently modulate Arabidopsis processes, leading to increased progeny production by this aphid, whereas orthologs of these effectors of $A$. pisum do not. However, the A. pisum effectors may modulate processes to the benefit of $A$. pisum in Fabaceae species. Indeed, $\mathrm{C} 002$ and PIntO1 were detected in A. pisum saliva (Carolan et al. 2009). C002 was also detected in the A. pisum plant hosts upon feeding (Mutti et al. 2008). Moreover, silencing of $\mathrm{C} 002$ in A. pisum is lethal to the aphid when it feeds from its plant host but not when aphids feed from an artificial diet (Mutti et al. 2006). At this time, we cannot exclude the possibility that PIntO2 is not functional in $A$. pisum, albeit this seems unlikely, given that we identified multiple full-lengths EST encoding this protein in A. pisum and other aphid species and all have predicted signal peptide sequences with high probability scores.

The positive effects on aphid reproduction were particularly profound on Arabidopsis plant lines that have relatively high aphid effector transgene expression levels, indicating that ample amounts of protein effectors are required for a beneficial

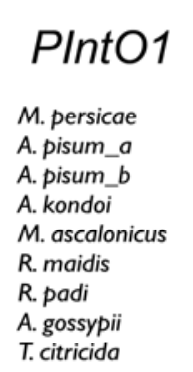

M. persicae

A. pisum_a

A. pisum_b

A. kondoi

M. ascalonicus

R. maidis

R. padi

A. gossypii

T. citricida

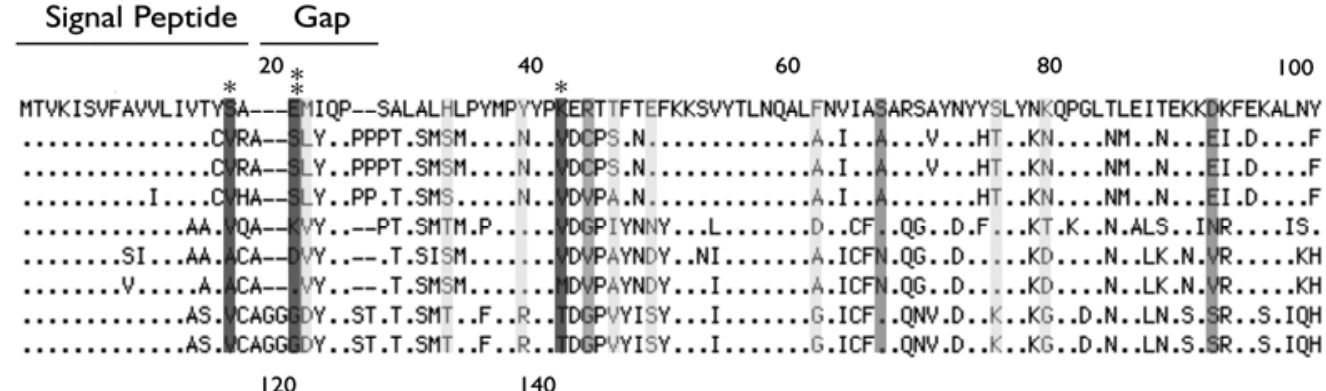

FENKL MDYRHSLIQFKTL INYDTNIPUTSLDDDIPIDRAIL ......K..QA...NY.A.LE.SN.S..ITV........EL. ..... KK.HQA..NY.A.LE.NN.SQ.ITV........EL. .....LK..QA..N. .A.LE.NKIT.IL.M.........DL. YDD..CI . .QE.LKLR, .LGCQ.APQPI ....N.S...DL. .DD.MNE..QA.LNY.L.LG.KSQV.-V....N.N..PEL. .DD.MNE..QA.LNY . M.LG.KNQM.-V ....N....PDL. .DE. .SE . .QA.MNY.L.LG.TN.NIPI ....N.G..SNL. .DE..SE..QA.MNY.L.LG.TN.NIPI ....N.G..SNL.

Fig. 7. Specific sites of PIntO1 are subject to positive selection. PIntO1 orthologs of various aphid species were aligned in Muscle (v3.7). Sites with high probability $(P>95 \%)$ of being under positive selection are highlighted in darkest gray, with $* *$ indicating $P>99 \%$. Sites with lower probabilities are highlighted in gray $(70 \%>P<95 \%)$ and light gray $(50 \%>P<70 \%)$. 
effect on aphid performance. This is in agreement with the abundance of these effectors in $M$. persicae and A. pisum saliva and the high expression levels of corresponding genes in these aphids. Whereas the expression levels of C002 and PIntO1 genes are higher than that of PIntO2, that of PIntO2 is nonetheless similar to that of $\beta$-tubulin, which is an abundant protein in aphids. C002, PIntO1, and PIntO2 transcripts were predominantly detected in $M$. persicae salivary glands (Bos et al. 2010) and C002 and PIntO1 have been identified as abundant proteins in $M$. persicae and $A$. pisum saliva (Carolan et al. 2009; Harmel et al. 2008). However, PIntO2 has not been identified in $M$. persicae and A. pisum saliva. Mass spectrometry may be biased toward the detection of the most abundant proteins. Furthermore, because saliva used for mass spectrometry is collected from aphids fed on diet, it is also possible that aphids produce PIntO2 more abundantly when feeding from plants but not when feeding from diet. Therefore. this study demonstrates that a functional genomics approach is complementary to other approaches used for the identification of aphid protein effectors, because it can result in the discovery of less abundant protein effectors in aphids or effectors that are specifically produced by aphids during plant feeding.

We previously demonstrated that silencing of rack- 1 and COO2 in $M$. persicae by plant-mediated RNAi leads to a reduction in M. persicae progeny on plants (Pitino et al. 2011). In this study, we demonstrated that plant-mediated RNAi of PIntO2 also reduces $M$. persicae reproduction to a similar degree as c002-silenced aphids on both $N$. benthamiana and Arabidopsis hosts. On the other hand, we did not observe an effect on progeny production of PIntOl-silenced $M$. persicae. It is possible that PIntO1 is redundant in function. Effectors may act redundantly by modifying the same plant targets or by modulating different processes in the same plant pathway (Cunnac et al. 2012). Conversely, C002 and PIntO2 may target more unique plant processes. Silencing of PIntO1 may also cause another as-yet-unknown phenotype in the aphid.

The phylogenetic tree of aphid species based on 203 concatenated nuclear genes (Ollivier et al. 2012) and those of the three protein effectors are similar. Unlike some microbial effectors (Liu et al. 2005; Win and Kamoun 2008; Win et al. 2007), C002, PIntO1, and PIntO2 do not belong to protein families. Thus, the protein effectors shared among the species are orthologs that have most likely diverged approximately 140 million years ago, when the extant aphid species diversified at the same time as main branches of the angiosperms (Von Dohlen and Moran 2000). The average $d_{N}$ versus $d_{S}$ nucleotide substitution rate of 253 reciprocal genes shared by aphid species ranges from 0.064 to 0.094 (Ollivier et al. 2010), which is much lower than that of the three effectors, which have pairwise $d_{N} / d_{S}$ values of 0.375 to 1.038 , indicating that effectors are fast evolving. Protein effectors are often subject to positive selection because of pressure to adapt to the their host targets that is under diversifying selection (Kaschani and Van der Hoorn 2011; Kaschani et al. 2010).

None of the ML models detected single sites under positive selection in C002. This is in agreement with Ollivier and associates (2010), who also found no significant evidence of positive selection of single sites in C002. However, the C002 alignment shows gap polymorphisms, which are excluded from model analyses to detect positively selected sites. We found that a mutant MpC002 in which the five NDNQGEE repeats are deleted does not promote $M$. persicae progeny production similarly to ApC002, which lacks the NDNQGEE repeat region, indicating that this region is essential for target specificity in Arabidopsis in relation to $M$. persicae. In addition, we found a second $M$. persicae $\mathrm{C} 002$ transcript encoding a protein with four NDNQGEE repeats instead of five, and the gap region is also polymorphic in $\mathrm{C} 002$ ortholog of the melon and cotton aphid Aphis gossypii. This indicates that the positively selected sites conferring target specificity may be located in the alignment gaps of C002. The ML models identified at least three sites with high significance of being under positive selection in PIntO1, whereas none were detected in PIntO2. The PIntO1 alignment shows small gap polymorphisms of 2 to 3 amino acids, whereas the $\mathrm{PIntO} 2$ alignment has larger gap polymorphisms, particularly at the C-terminal ends of the proteins. Therefore, it is possible that sites in alignments gaps of PIntO2 are under positive selection, similarly to $\mathrm{C} 002$.

In summary, we have provided evidence that $M$. persicae effectors modulate Arabidopsis processes that are advantageous to $M$. persicae colonization ability. In contrast, the Acyrthosiphon pisum orthologs appear not functional in Arabidopsis in relation to $M$. persicae colonization. This is in agreement with the host ranges of these two aphid species, with $M$. persicae being able to readily feed and reproduce on Arabidopsis and A. pisum having a host range predominantly restricted to the Fabaceae family and incapable of colonizing Arabidopsis. We also provide evidence that aphid effectors are fast evolving, in which specific sites under selection were identified in PIntO1 and those in $\mathrm{C} 002$ and $\mathrm{PIntO} 2$ may be located in alignment gaps. Evidence for the latter is provided for $\mathrm{C} 002$, because an $M$. persicae $\mathrm{C} 002$ mutant without the NDNQGEE repeat region, which overlaps with an alignment gap in $\mathrm{C} 002$, does not promote $M$. persicae colonization on Arabidopsis. Taken together, these results provide evidence that aphid effectors contribute to aphid-plant compatibility.

\section{MATERIALS AND METHODS}

\section{Insect rearing.}

The aphid lineages used in this study are $M$. persicae RRes (Clone O) (Bos et al. 2010) and A. pisum DbS (collected from Pisum sativum field crop near Dickleborough, Suffolk in 2005). $M$. persicae were reared on $N$. tabacum or $N$. benthamiana plants for $N$. benthamiana leaf disk assays and on Chinese cabbage (Brassica rapa) for the fecundity assays on Arabidopsis thaliana. A. pisum were reared on Vicia faba plants. The insects were maintained in custom-built acrylic cages located in controlled-environment conditions at $18^{\circ} \mathrm{C}$ under $16 \mathrm{~h}$ of light.

\section{Aphid cDNA synthesis.}

Total RNA was prepared from 5 to 10 aphids. Liquid nitrogen frozen samples were homogenized for $60 \mathrm{~s}$ to a fine powder using a Mini-Bead-Beater-8TM (Biospec Products, Bartlesville, OK, U.S.A.) and 1.2-mm stainless steel beads. After the homogenization, the samples were used for RNA extraction with TRI Reagent (Sigma-Aldrich, St. Louis) and purified using Qiagen RNeasy columns (Qiagen Ltd., West Sussex, U.K.). cDNA was synthesized from $0.5 \mu \mathrm{g}$ of total RNA using Moloney murine leukemia virus (M-MLV) reverse transcriptase (Invitrogen, Carlsbad, CA, U.S.A.). Samples were then stored at $-80^{\circ} \mathrm{C}$.

\section{Generation of constructs}

\section{for phloem expression or dsRNA constructs.}

To generate transgenic Arabidopsis, coding nucleotide sequences for PIntO1 and PIntO2 without the region corresponding to the signal peptides were codon optimized for efficient expression in Arabidopsis thaliana and cloned into pUC57 vector (Genscript, Piscataway, NJ, U.S.A.). These sequences were amplified with specific primers with additional attb1 and attb2 linker sites for cloning into the plasmid vector pDONR207 (Invitrogen) plasmid using Gateway BP reaction and trans- 
formed into $\mathrm{DH} 5 \alpha$. Coding sequences without a signal peptide sequence for $\mathrm{C} 002$ were directly amplified from aphid total RNAs by reverse-transcriptase polymerase chain reaction (RTPCR) using specific primers containing additional attb1 and attb2 linker sites (Supplementary Table S7). MpC002 $\Delta$ was generated using an overlapping PCR approach using the MpC002_a sequence as template and with specific primers. The GFP open reading frame was amplified with specific primers. All clones were sequenced to verify correct size and sequence of inserts. Next, the inserts were introduced into the destination vector pB7WG2 carrying the AtSuc2 promoter sequence for phloemspecific expression (Gottwald et al. 2000) using the Gateway LR reaction procedure. The vectors were introduced into Agrobacterium tumefaciens GV3101 plasmid and used for generating transgenic Arabidopsis thaliana as described previously (Pitino et al. 2011).

To generate the dsRNA constructs, a 710-bp MpC002_a fragment corresponding to the entire mature $M$. persicae MpC002_a protein without the signal peptide (as described previously) (Pitino et al. 2011), a 263-bp MpPIntO1 fragment starting at nucleotide position +69 (CCGTCCG and ending at nucleotide position +332 (CGTTGAT) of the $M$. persicae PIntO1 transcript sequence, a 254-bp PIntO2 fragment starting at nucleotide position +26 (CGTGATA) and ending at nucleotide position +280 (TCAC-CGG) of the M. persicae $\mathrm{PIntO} 2$ transcript sequence, and a 537-bp GFP fragment starting at nucleotide position +29 (GAGTGG) and ending at nucleotide position +566 (...TTAGCAG) of the GFP open reading frame (as described previously) (Pitino et al. 2011) were introduced into pDONR207 (Invitrogen) plasmid using Gateway BP reaction and transformed into DH5 $\alpha$. Subsequent clones were sequenced to verify correct size and sequence of inserts. Subsequently, the inserts were introduced into the pJawohl8-RNAi binary silencing vector (I. E. Somssich, Max Planck Institute) using Gateway LR reaction generating plasmids pJMpC002 and pJGFP (Pitino et al. 2011) and pJAMpPIntO1 and pJAMpPIntO2 (this study). The four constructs were introduced into Agrobacterium tumefaciens GV3101 containing pMP90RK plasmid and used for transient assays in $N$. benthamiana leaves and transformation of Arabidopsis thaliana.

\section{Generation of transgenic plants.}

Arabidopsis thaliana Col-0 plants were grown in a growth chamber under long-day conditions until flowering. Flowers were dipped in an Agrobacterium tumefaciens (GV3101) suspension containing the appropriate plasmid. T1 seed were germinated in soil and the transformants were BASTA selected. T2 seed of BASTA-resistant lines were plated on MurashigeSkoog media containing phosphinothricin at $20 \mu \mathrm{g} / \mathrm{ml}$. The lines showing a single insertion based on a 3:1 segregation ratio of live/dead seedlings were selected, and homozygous progeny of these plants were used for further analyses.

\section{Arabidopsis RNA extraction.}

Total RNA was prepared from 3- to 6-week-old plant materials. Liquid-nitrogen-frozen leaves were homogenized for $60 \mathrm{~s}$ to a fine powder using a Mini-Bead-Beater-8TM (Biospec Products) and 1.2-mm stainless steel beads. After the homogenization, the samples were used for RNA extraction with TRI Reagent (Sigma-Aldrich) and purified using Qiagen RNeasy columns (Qiagen Ltd.). cDNA was synthesized from $0.5 \mu \mathrm{g}$ of total RNA using M-MLV reverse transcriptase (Invitrogen). Samples were then stored at $-80^{\circ} \mathrm{C}$.

\section{RT-quantitative PCR analysis.}

Total RNA was extracted from Arabidopsis thaliana leaves or aphids using TRIzol reagent. DNA contaminations were removed by treating RNA extraction with RNase-free DNase (Qiagen Ltd.) and purified with QIAamp columns (Qiagen Ltd.). First-strand cDNA was synthesized at $37^{\circ} \mathrm{C}$ from total RNA using M-MLV (Invitrogen) reverse transcriptase according to the manufacturer's instructions and subjected to realtime PCR using SYBR Green JumpStart Taq ReadyMix (Sigma-Aldrich) in a DNA Engine Opticon 2 (Bio-Rad, Life Sciences) using gene-specific primers. For RT-quantitative (q)PCR of Arabidopsis, the cycle threshold (Ct) values were normalized using Arabidopsis actin as reference (Sugio et al. 2011). For RT-qPCR in aphids, the $\mathrm{Ct}$ values were normalized for difference in cDNA amount using tubulin and L27 transcripts as reference (Mutti et al. 2006; Shakesby et al. 2009). Fold changes were calculated by comparing the normalized transcript levels of $\mathrm{C} 002$, PIntO1, and $\mathrm{PIntO} 2$ of $M$. persicae fed on C002-dsRNA, PIntO1-dsRNA, and PIntO2-dsRNA transgenic plants to aphids fed on GFP-dsRNA transgenic plants, as previously described (Pitino et al. 2011).

\section{M. persicae fecundity assay on Arabidopsis transgenic lines.}

Three transgenic Arabidopsis T3 lines for each construct (AtSUC2-) were used for each experiment, with wild-type Col-0 plants and AtSUC2:GFP plants as controls. To measure the $M$. persicae fecundity, four plants each from independent transgenic and wild-type lines were grown for 4 weeks in a controlled environment room in pots with soil (type of soil: Levington F2), and maintained under conditions of $18^{\circ} \mathrm{C}$ (day) and $16^{\circ} \mathrm{C}$ (night), $8 \mathrm{~h}$ of light, and $60 \%$ humidity. The seed were cold treated for 2 days in a refrigerator $\left(4^{\circ} \mathrm{C}\right)$ in order to synchronize germination. After 4 weeks, the plants were moved to new pots (one plant per pot) with soil (F2) and covered by a transparent plastic cover with mesh on the top to allow plant transpiration and prevent insect escape. For $M$. persicae fecundity assays, five adult aphids were transferred from the host Chinese cabbage ( $B$. rapa subsp. chinensis) plants to 4-week-day old Arabidopsis plants within the sealed experimental cages. Two days later, five nymphs were left on the plants and the adults were removed. Numbers of offspring produced on each plant at the 10th, 13th, and 16th day of the experiment were counted and removed.

\section{N. benthamiana leaf infiltration and leaf disk assays.}

Single Agrobacterium colonies harboring pJGFP, pJMpC002, pJAMpPIntO1, and pJAMpPIntO2 were inoculated into Luria broth containing kanamycin at $25 \mathrm{mg} / \mathrm{liter}$, gentamicin at 25 $\mathrm{mg} / \mathrm{liter}$, rifampicin at $50 \mathrm{mg} / \mathrm{liter}$, and carbenicillin at 25 $\mathrm{mg} /$ liter and grown $\left(28^{\circ} \mathrm{C}\right.$ at $\left.225 \mathrm{rpm}\right)$ until an optical density at $600 \mathrm{~nm}$ of 0.3 was reached (Eppendorf BioPhotometer; Eppendorf, Cambridge, U.K.). Cultures were resuspended in infiltration medium $(10 \mathrm{mM} \mathrm{MgCl}$ and $10 \mathrm{mM}$ 2-(N-morpholino)ethanesulfonic acid, $\mathrm{pH}$ 5.6) with $150 \mathrm{mM}$ acetosyringone to initiate expression. Each construct was infiltrated into the youngest fully expanded leaves of 4-to 6-week-old $N$. benthamiana plants. The plants were grown in a growth chamber with daily temperatures of 22 to $25^{\circ} \mathrm{C}$ under a short-day regime. One day after infiltration, leaves were harvested and used in leaf disk assays. The leaf disks were cut from the infiltrated areas using an 11-mm-diameter borer and placed in single wells of a 24-well plate on top of a plug consisting of $1 \mathrm{ml}$ of solidified $1 \%$ distilled water agar. Four first-instar nymphs (1 to 2 days old) reared on $N$. tabacum were placed onto the leaf disks for a total of six leaves per construct. The wells were individually sealed with mesh lids and put upside down in controlled-environment conditions at $18^{\circ} \mathrm{C}$ under $16 \mathrm{~h}$ of light. The 24-well plate was replaced with freshly infiltrated (1 day post infiltration) leaf disks after 6 and 12 days. Aphid survival was counted at $6,12,14$, and 17 days after initial transfer of 
aphids to the first 24-well plate, and the numbers of nymphs produced by these aphids at 12,14 , and 17 days were also counted. The nymphs were removed after counting. This experiment was repeated six times to generate six independent biological replicates, each containing six leaf-disks per construct.

\section{Statistical analysis}

\section{of $M$. persicae survival and reproduction data.}

All statistical analyses were conducted using the GenStat 11 statistical package (VSNi Ltd., Hemel Hempstead, U.K.) as previously described (Pitino et al. 2011) and is here described for clarity. Data were checked for approximate normal distribution by visualizing residuals. Classical linear regression analysis using a GLM with Poisson distributions was applied to analyze the $M$. persicae fecundity on plants, with "nymphs" as a response variable. The aphid nymph production on four plants per treatment were used as independent data points in statistical analyses in which the biological replicate was used as a variable.

$N$. benthamiana leaf disk assay fecundity data were analyzed using an unbalanced one-way ANOVA design, with "construct" as the treatment and "repeat" as the block. In the $N$. benthamiana leaf disk assay, aphid fecundity was monitored on individual leaf disks at six disks per treatment. Numbers of aphid nymphs produced on each leaf disk were used as independent data points in statistical analyses in which the biological replicate was used as a variable. Leaf disks that dried up because of lack of humidity were excluded, giving four to six leaf disks per construct for each biological replicate.

\section{Positive selection analysis.}

M. persicae $\mathrm{C} 002$, $\mathrm{PIntO} 1$, and $\mathrm{PIntO} 2$ nucleotide sequences were searched against the EST databases of GenBank using blastn (Altschul et al. 1990). All the similar sequences were downloaded and were aligned using Muscle (Edgar 2004a and b) in CLC Main Workbench (Aarhus, Denmark). Incomplete EST were removed and remaining sequences were analyzed for start and stop codons to ensure their full lengths.

Nucleotide sequences of full-length EST were aligned in Muscle (v3.7) and saved in PHYLIP format. The phylogenetic tree was reconstructed with remove gaps enabled and using the ML method implemented in the PhyML program (v3.0 aLRT). The GTR substitution model was selected with 100 bootstrap replicates. Estimates of $d_{N}$ versus $d_{S}$ were computed pairwise between sequences using the approximate methods (Nei and Gojobori 1986). Maximum-likelihood (ML) analysis of codon substitutions were performed under different models with the program CODEML implemented in the PAML 4.4 software package (Goldman and Yang 1994; Yang and Bielawski 2000). The different models were compared using the likelihood ratio test (LRT), which compares twice the differences in log likelihood to a $\chi^{2}$ distribution with the required number of degrees of freedom. The six models recommended by Yang and Bielawski (2000) were tested. They were null models M0 (one ratio), $\mathrm{M} 1$ (neutral), and $\mathrm{M} 7(\beta)$, corresponding to alternative models M3 (discrete), M2 (selection), and M8 $(\beta+\omega)$, respectively. (Nielsen and Yang 1998; Yang and Bielawski 2000). Significant differences between the two models were evaluated by calculating the LRT following $\chi^{2}$ distribution. We use an LRT comparing M0 (one ratio) with M3 (discrete) to test for variable selective pressure among sites, and LRT to test for sites evolving by positive selection, comparing M1 (neutral) versus M2 (selection) and M7 $(\beta)$ against M8 $(\beta+\omega)$. When LRT was significant, we used Bayes Empirical Bayes analysis for detection of positive sites (Nielsen and Yang 1998; Yang and Bielawski 2000).

\section{ACKNOWLEDGMENTS}

This work was supported by BB/J004553/1 from Biotechnology and Biological Sciences Research Council (BBSRC) and the John Innes Foundation. We thank D. Prince, A. Coleman, and M. Maffei for carefully reading the manuscript; and the insectary staff at the John Innes Centre for rearing the insects used in this study.

\section{LITERATURE CITED}

Altschul, S. F., Gish, W., Miller, W., Myers, E. W., and Lipman, D. J. 1990. Basic local alignment search tool. J. Mol. Biol. 215:403-410.

Bos, J. I., Prince, D., Pitino, M., Maffei, M. E., Win, J., and Hogenhout, S. A. 2010. A Functional genomics approach identifies candidate effectors from the aphid species Myzus persicae (green peach aphid). PLoS Genet. 6:e1001216. Published online.

Carolan, J. C., Fitzroy, C. I., Ashton, P. D., Douglas, A. E., and Wilkinson, T. L. 2009. The secreted salivary proteome of the pea aphid Acyrthosiphon pisum characterised by mass spectrometry. Proteomics 9:24572467.

Carolan, J. C., Caragea, D., Reardon, K. T., Mutti, N. S., Dittmer, N., Pappan, K., Cui, F., Castaneto, M., Poulain, J., Dossat, C., Tagu, D., Reese, J. C., Reeck, G. R., Wilkinson, T. L., and Edwards, O. R. 2011. Predicted effector molecules in the salivary secretome of the pea aphid (Acyrthosiphon pisum) —A dual transcriptomic-proteomic approach. J. Proteome Res. 10:1505-1518.

Cherqui, A., and Tjallingii, W. F. 2000. Salivary proteins of aphids, a pilot study on identification, separation and immunolocalisation. J. Insect Physiol. 46:1177-1186.

Cunnac, S., Chakravarthy, S., Kvitko, B. H., Russell, A. B., Martin, G. B., and Collmer, A. 2012. Genetic disassembly and combinatorial reassembly identify a minimal functional repertoire of type III effectors in Pseudomonas syringae. Proc. Natl. Acad. Sci. U.S.A. 108:2975-2980.

Dogimont, C., Bendahmane, A., Chovelon, V., and Boissot, N. 2010. Host plant resistance to aphids in cultivated crops: Genetic and molecular bases, and interactions with aphid populations. C. R. Biol. 333:566-573.

Edgar, R. C. 2004a. MUSCLE: Multiple sequence alignment with high accuracy and high throughput. Nucleic Acids Res. 32:1792-1797.

Edgar, R. C. 2004b. MUSCLE: A multiple sequence alignment method with reduced time and space complexity. BMC Bioinf. 5:113.

Goldman, N., and Yang, Z. 1994. A codon-based model of nucleotide substitution for protein-coding DNA sequences. Mol. Biol. Evol. 11:725-736.

Gottwald, J. R., Krysan, P. J., Young, J. C., Evert, R. F., and Sussman, M. R. 2000. Genetic evidence for the in planta role of phloem-specific plasma membrane sucrose transporters. Proc. Natl. Acad. Sci. U.S.A. 97:13979-13984.

Harmel, N., Letocart, E., Cherqui, A., Giordanengo, P., Mazzucchelli, G., Guillonneau, F., De Pauw, E., Haubruge, E., and Francis, F. 2008. Identification of aphid salivary proteins: A proteomic investigation of Myzus persicae. Insect Mol. Biol. 17:165-174.

Hogenhout, S. A., and Bos, J. I. 2011. Effector proteins that modulate plant-insect interactions. Curr. Opin. Plant Biol. 14:422-428.

Hogenhout, S. A., Van der Hoorn, R. A., Terauchi, R., and Kamoun, S. 2009. Emerging concepts in effector biology of plant-associated organisms. Mol. Plant-Microbe Interact. 22:115-122.

IAGC. 2010. Genome sequence of the pea aphid Acyrthosiphon pisum. PLoS Biol. 8:e1000313. Published online.

Ina, Y. 1995. New methods for estimating the numbers of synonymous and nonsynonymous substitutions. J. Mol. Evol. 40:190-226.

Kaschani, F., and Van der Hoorn, R. A. 2011. A model of the C14-EPIC complex indicates hotspots for a protease-inhibitor arms race in the oomycete-potato interaction. Plant Signal. Behav. 6:109-112.

Kaschani, F., Shabab, M., Bozkurt, T., Shindo, T., Schornack, S., Gu, C., Ilyas, M., Win, J., Kamoun, S., and van der Hoorn, R. A. 2010. An effector-targeted protease contributes to defense against Phytophthora infestans and is under diversifying selection in natural hosts. Plant Physiol. 154:1794-1804.

Li, W. H., Wu, C. I., and Luo, C. C. 1985. A new method for estimating synonymous and nonsynonymous rates of nucleotide substitution considering the relative likelihood of nucleotide and codon changes. Mol. Biol. Evol. 2:150-174.

Liu, Z., Bos, J. I., Armstrong, M., Whisson, S. C., da Cunha, L., TortoAlalibo, T., Win, J., Avrova, A. O., Wright, F., Birch, P. R., and Kamoun, S. 2005. Patterns of diversifying selection in the phytotoxin-like scr74 gene family of Phytophthora infestans. Mol. Biol. Evol. 22:659-672.

Miles, P. 1999. Aphid saliva. Biol. Rev. 74:41-85.

Mutti, N. S., Park, Y., Reese, J. C., and Reeck, G. R. 2006. RNAi knockdown of a salivary transcript leading to lethality in the pea aphid, Acyrthosiphon pisum. J. Insect Sci. 6:1-7. 
Mutti, N. S., Louis, J., Pappan, L. K., Pappan, K., Begum, K., Chen, M.S., Park, Y., Dittmer, N., Marshall, J., Reese, J. C., and Reeck, G. R. 2008. A protein from the salivary glands of the pea aphid, Acyrthosiphon pisum, is essential in feeding on a host plant. Proc. Natl. Acad. Sci. U.S.A. 105:9965-9969.

Nei, M., and Gojobori, T. 1986. Simple methods for estimating the numbers of synonymous and nonsynonymous nucleotide substitutions. Mol. Biol. Evol. 3:418-426.

Nicholson, S. J., Hartson, S. D., and Puterka, G. J. 2012. Proteomic analysis of secreted saliva from Russian wheat aphid (Diuraphis noxia Kurd.) biotypes that differ in virulence to wheat. J. Proteomics 75:2252-2268.

Nielsen, R., and Yang, Z. 1998. Likelihood models for detecting positively selected amino acid sites and applications to the HIV-1 envelope gene. Genetics 148:929-936.

Ollivier, M., Legeai, F., and Rispe, C. 2010. Comparative analysis of the Acyrthosiphon pisum genome and expressed sequence tag-based gene sets from other aphid species. Insect Mol. Biol. 19 (Suppl. 2):33-45.

Ollivier, M., Gabaldon, T., Poulain, J., Gavory, F., Leterme, N., Gauthier, J. P., Legeai, F., Tagu, D., Simon, J. C., and Rispe, C. 2012. Comparison of gene repertoires and patterns of evolutionary rates in eight aphid species that differ by reproductive mode. Genome Biol. Evol. 4:155167

Pitino, M., Coleman, A. D., Maffei, M. E., Ridout, C. J., and Hogenhout, S. A. 2011. Silencing of aphid genes by dsRNA feeding from plants. PLoS One 6:e25709. Published online.

Ramsey, J. S., Wilson, A. C., de Vos, M., Sun, Q., Tamborindeguy, C., Winfield, A., Malloch, G., Smith, D. M., Fenton, B., Gray, S. M., and Jander, G. 2007. Genomic resources for Myzus persicae: EST sequencing, SNP identification, and microarray design. BMC Genomics 8:423.

Schoonhoven, L., van Loon, J. J., and Dicke, M. 2005. Insect-Plant Biology. Oxford University Press, Oxford.
Shakesby, A. J., Wallace, I. S., Isaacs, H. V., Pritchard, J., Roberts, D. M and Douglas, A. E. 2009. A water-specific aquaporin involved in aphid osmoregulation. Insect Biochem. Mol. Biol. 39:1-10.

Sugio, A., Kingdom, H. N., MacLean, A. M., Grieve, V. M., and Hogenhout, S. A. 2011. Phytoplasma protein effector SAP11 enhances insect vector reproduction by manipulating plant development and defense hormone biosynthesis. Proc. Natl. Acad. Sci. U.S.A. 108:E1254-1263.

Tian, D., Peiffer, M., Shoemaker, E., Tooker, J., Haubruge, E., Francis, F., Luthe, D. S., and Felton, G. W. 2012. Salivary glucose oxidase from caterpillars mediates the induction of rapid and delayed-induced defenses in the tomato plant. PLoS One 7:e36168. Published online.

Tjallingii, W. 2006. Salivary secretions by aphids interacting with proteins of phloem wound responses. J. Exp. Bot. 57:739-745.

Von Dohlen, C. D., and Moran, N. A. 2000. Molecular data support a rapid radiation of aphids in the Cretaceous and multiple origins of host alternation. Biol. J. Linn. Soc. Lond. 71:689-717.

Will, T., Tjallingii, W. F., Thonnessen, A., and van Bel, A. J. E. 2007. Molecular sabotage of plant defense by aphid saliva. Proc. Natl. Acad. Sci. U.S.A. 104:10536-10541.

Will, T., Kornemann, S. R., Furch, A. C., Tjallingii, W. F., and van Bel, A. J. 2009. Aphid watery saliva counteracts sieve-tube occlusion: A universal phenomenon? J. Exp. Biol. 212:3305-3312.

Win, J., and Kamoun, S. 2008. Adaptive evolution has targeted the C-terminal domain of the RXLR effectors of plant pathogenic oomycetes. Plant Signal. Behav. 3:251-253.

Win, J., Morgan, W., Bos, J., Krasileva, K. V., Cano, L. M., Chaparro-Garcia, A., Ammar, R., Staskawicz, B. J., and Kamoun, S. 2007. Adaptive evolution has targeted the C-terminal domain of the RXLR effectors of plant pathogenic oomycetes. Plant Cell 19:2349-2369.

Yang, Z., and Bielawski, J. P. 2000. Statistical methods for detecting molecular adaptation. Trends Ecol. Evol. 15:496-503. 